
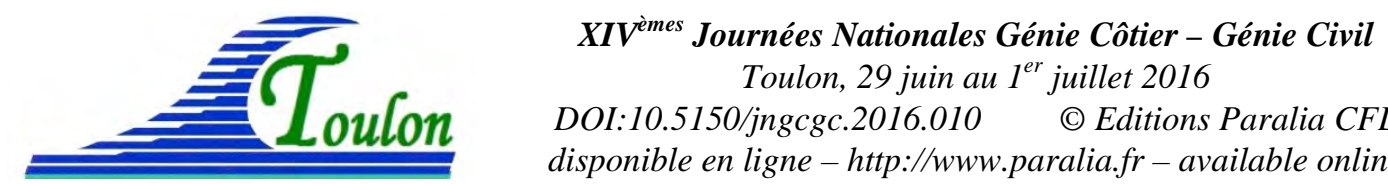

DOI:10.5150/jngcgc.2016.010 $\quad$ (C) Editions Paralia CFL

disponible en ligne - http://www.paralia.fr - available online

\title{
Analyse de l'impact physique d'une souille de dragage sur la stabilité du littoral
}

\author{
Mimoun OUKADR ${ }^{1}$, Mohamed CHAGDALI ${ }^{1}$, Soumia MORDANE ${ }^{1}$ \\ 1. Université Hassan II Casablanca - Faculté des Sciences Ben M’Sik - LPPPM, \\ Casablanca, Maroc.oukadr@gmail.com
}

\section{Résumé :}

On se propose d'analyser par modélisation numérique l'impact d'une dépression du fond (souille) due au dragage sur l'altération des caractéristiques de la houle : Hauteurs, direction. L'objectif est d'apporter un élément technique pour mieux analyser l'impact de dragage sur l'environnement sur son volet physique. On analyse la configuration de référence du site d'étude avant et après la modification du fond par dragage.

L'étude est faite sur un site réel qui se trouve sur la façade atlantique Marocaine. Le modèle hydrodynamique utilisé est le modèle numérique de propagation de houle "SWAN" développé par l'université technologique de Delft. Il est adapté à la propagation de houle en zone côtière. Les résultats présentés sont relatifs aux variations de l'énergie et de la direction avant et après extraction du sable.

Mots-clés : Etude d'impact sur l'environnement, Modélisation de la propagation de la houle par SWAN, Impact d'une souille de dragage sur la stabilité du littoral.

\section{Introduction}

Le dragage manifestera un impact direct sur la bathymétrie et sur la morphologie des fonds marins du site (MIGNIOT \& VIGUIER, 1980). La modification de la bathymétrie a une conséquence directe sur la propagation de la houle dès lors que celle-ci présente une longueur d'onde suffisante pour être sensible aux fluctuations du fond (MIGNIOT, 1990). Ce problème a fait l'objet de plusieurs études et projets. On cite les travaux de TING et RAICHLEN (1986) sur l'interaction de la houle avec une souille rectangulaire. Le projet Européen EU FP5 Sandpit piloté par Delft Hydraulics et qui a été centré sur la problématique des souilles d'extraction de granulats. KOJIMA et al. (1986) a constaté des érosions sur le littoral de la mer Genkai au Japon suite à la présence des fosses de dragage dans des profondeurs moins de $30 \mathrm{~m}$. BENDER (2001) a mené aussi des expérimentations en laboratoire pour constater l'impact des fosses sur la ligne de côte. DEMIR et al. (2004) a étudié via une modélisation numérique le comportement de la houle au-dessus d'une fosse de dragage à Istanbul en mer noire. Une revue des travaux relatifs aux techniques de dragage est présentée par un consortium Européen au journal JCR (VELEGRAKIS et al., 2010). Dans cette étude, l'objectif est d'avoir un outil numérique d'évaluation de l'impact hydrodynamique des travaux de dragage dans la zone du site. La notion de l'impact hydrodynamique est définie par l'altération du 


\section{Thème 1 - Hydrodynamique côtière}

champ de vagues par la dépression et les conséquences que cela engendre sur les enjeux à la côte. Cet outil est testé dans une configuration réelle avec un environnement naturel. Le modèle hydrodynamique utilisé est le modèle numérique de propagation de houle "SWAN" développé par l'université technologique de Delft (BOOIJ et al., 1999). Il est adapté à la propagation de houle en zone côtière et est très largement exploité. Dans ce travail, le site de référence est celui de Casablanca qui se trouve sur la façade atlantique du Maroc. En considérant les volumes à extraire au cours des prochaines années d'exploitation il est possible d'estimer l'approfondissement théorique que devrait connaître la zone, en envisageant le cas d'une exploitation parfaitement uniforme sur toute la superficie de la fosse. L'approfondissement théorique moyen sur l'ensemble du périmètre serait de $5 \mathrm{~m}$ environ. Dans la réalité, cet approfondissement est moindre contenu du comblement par les sédiments issus du transport sédimentaire par transit littoral dans le proche littoral.

\section{Présentation du site d'étude}

Le littoral Casablanca, portion du littoral atlantique marocain, est situé à l'Ouest du Maroc. La zone côtière Casablanca - Mohammedia s'étend sur environ $20 \mathrm{Km}$ et englobe le secteur littoral situé entre le port de Casablanca et le port de Mohammedia. Le tracé de la côte du littoral présente comme le reste du littoral atlantique marocain de nombreuses plages sableuses, des falaises rocheuses et des platiers rocheux. L'examen de la carte bathymétrique du plateau continental de la région proposé montre que la morphologie du fond est assez variée.

Les données suivantes ont été extraites des prévisions et analyses océanométéorologiques réalisées par la Direction de la Météorologie Nationale du Maroc. Ces données possèdent un degré de fiabilité important et sont régulièrement exploitées pour des études d'impact.

\subsection{La houle}

La détermination de la climatologie au large du site a été effectuée à partir de la base de données SIMAR 1048036 qui, par reconstitution des champs de vent, couvre 44 années de données à raison d'une donnée toutes les 3 heures et validées sur la côte atlantique Marocaine par comparaison à des mesures de bouées disponibles.

Le tableau 1 présente la répartition moyenne des houles (exprimée en \%) par classe de hauteur et par direction de provenance de la houle.

On note que la houle dominante provient du secteur $310^{\circ} \mathrm{N}$ à $340^{\circ} \mathrm{N}$, qui regroupe la majorité des occurrences de houle. Par ailleurs, les houles les plus fortes proviennent du secteur $290^{\circ} \mathrm{N}$ à $310^{\circ} \mathrm{N}$. 


\section{XIV èmes Journées Nationales Génie Côtier - Génie Civil \\ Toulon, 29 juin au $1^{\text {er }}$ juillet 2016}

\subsection{Les marées}

Les masses d'eaux marines de la côte atlantique marocaine sont soumises à une onde de marée de type semi-diurne régulière avec deux pleines-mers (PM) et deux basses-mers (BM) journalières de hauteurs respectives sensiblement égales et séparés, en moyenne avec une période de $12 \mathrm{~h} 25 \mathrm{mn}$. On enregistre un marnage moyen de 2,19 m, un maximum de 3,54 m environ lors de vives eaux et un minimum de 0,86 lors de mortes eaux.

Tableau 1. Régime de houle moyen- répartition Hs/Dir en \%.

\begin{tabular}{|c|c|c|c|c|c|c|c|c|c|c|c|c|c|c|c|c|c|c|}
\hline & \multicolumn{16}{|c|}{ Directions moyennes des houles $\left({ }^{\circ} \mathrm{N}\right)$} & & \\
\hline & $\mathbf{N}$ & NNE & NE & ENE & $\mathbf{E}$ & ESE & SE & SSE & $\mathbf{S}$ & SSW & SW & WSW & $\mathbf{w}$ & WNW & NW & NNW & & \\
\hline $\mathrm{Hs}(\mathrm{m})$ & 0 & 22,5 & 45 & 67,5 & 90 & 112,5 & 135 & 157,5 & 180 & 203 & 225 & 248 & 270 & 292,5 & 315 & 337,5 & Total en \% & classes de $\mathrm{Hs}$ \\
\hline $0-0.5$ & & & & & & & & & & & & & 0,02 & 0,148 & 0,1 & 0,039 & 0,31 & $0-0.5$ \\
\hline $0.5-1$ & 0,076 & & & & & & & & & & & & 0,29 & 1,86 & 7,49 & 5,411 & 15,12 & $0.5-1$ \\
\hline $1-1.5$ & 0,14 & & & & & & & & & & & & 0,49 & 2,49 & 11,7 & 20,22 & 34,99 & $1-1.5$ \\
\hline $1.5-2$ & 0,072 & & & & & & & & & & & & 0,22 & 1,61 & 8,7 & 11,7 & 22,30 & $1.5-2$ \\
\hline $2-2.5$ & 0,015 & & & & & & & & & & & & 0,05 & 0,8 & 6,85 & 5,76 & 13,48 & $2-2.5$ \\
\hline $2.5-3$ & 0,006 & & & & & & & & & & & & $O$ & 0,4 & 2,74 & 2,95 & 6,10 & $2.5-3$ \\
\hline $3-3.5$ & & & & & & & & & & & & & & 0,198 & 1,54 & 1,56 & 3,30 & $3-3.5$ \\
\hline $3.5-4$ & 0,001 & & & & & & & & & & & & & 0,09 & 0,92 & 0,858 & 1,87 & $3.5-4$ \\
\hline $4-4.5$ & & & & & & & & & & & & & & 0,054 & 0,58 & 0,496 & 1,13 & $4-4.5$ \\
\hline $4.5-5$ & & & & & & & & & & & & & & 0,018 & 0,31 & 0,243 & 0,57 & $4.5-5$ \\
\hline $5-5.5$ & & & & & & & & & & & & & & 0,004 & 0,18 & 0,162 & 0,34 & $5-5.5$ \\
\hline $5.5-6$ & & & & & & & & & & & & & & 0,001 & 0,12 & 0,077 & 0,20 & $5.5-6$ \\
\hline $6-6.5$ & & & & & & & & & & & & & & & 0,08 & 0,041 & 0,12 & $6-6.5$ \\
\hline $6.5-7$ & & & & & & & & & & & & & & & 0,06 & 0,019 & 0,08 & $6.5-7$ \\
\hline $7-7.5$ & & & & & & & & & & & & & & & 0,03 & 0,007 & 0,04 & $7-7.5$ \\
\hline $7.5-8$ & & & & & & & & & & & & & & & 0,02 & 0,005 & 0,03 & $7.5-8$ \\
\hline $8-8.5$ & & & & & & & & & & & & & & & 0,02 & 0,003 & 0,02 & $8-8.5$ \\
\hline $8.5-9$ & & & & & & & & & & & & & & & 0,01 & 0,002 & 0,01 & $8.5-9$ \\
\hline $9-9.5$ & & & & & & & & & & & & & & & 0,01 & & 0,01 & $9-9.5$ \\
\hline $9.5-10$ & & & & & & & & & & & & & & & & & & $9.5-10$ \\
\hline $10-11$ & & & & & & & & & & & & & & & & & & $10-11$ \\
\hline Total en \% & 0,31 & 0 & 0 & 0 & 0 & 0 & 0 & 0 & 0 & 0 & 0 & 0 & 1,07 & 7,673 & 41,4 & 49,55 & 100,00 & \\
\hline
\end{tabular}

\subsection{Evolution du trait de côte}

Les travaux de (IDRISSI et al., 2004) montre que certaines plages subissent un recul, tandis que d'autres subissent une avancée (figure 1).

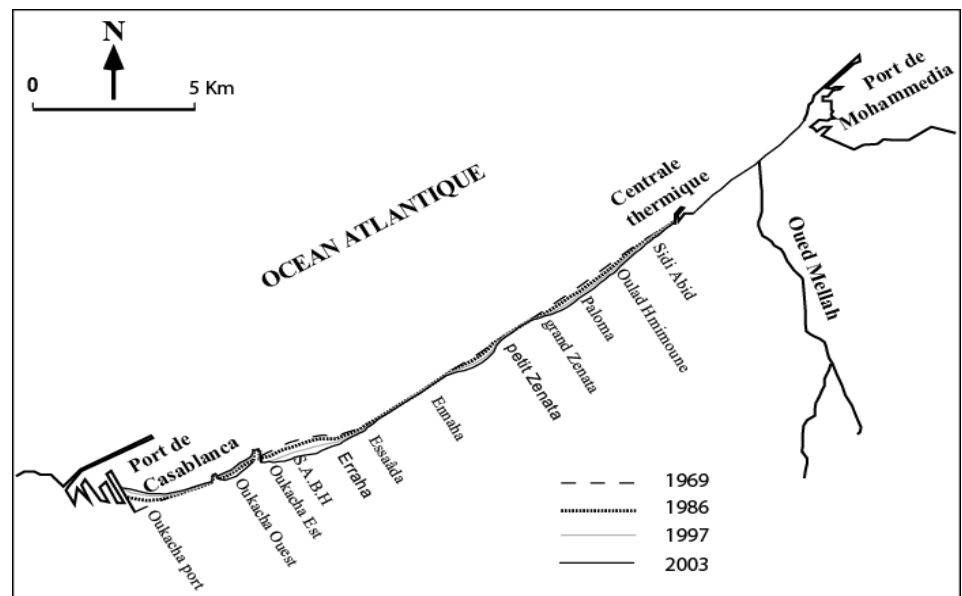

Figure 1. Evolution du trait de côte de la zone côtière Casablanca-Mohammedia.

Pour le site de Casablanca, la houle est principalement une houle formée au large. Le long des côtes du Maroc, la houle n'a généralement pas de rapport avec le temps qu'il 


\section{Thème 1 - Hydrodynamique côtière}

fait sur la côte. Elle est, le plus souvent, la conséquence des dépressions barométriques passant entre l'Archipel des Açores et l'Islande.

En égard à l'objectif principal de cette étude les effets de vent n'ont pas été pris en compte. Le phénomène de houle formée est jugé prépondérant par rapport à l'objectif d'analyse d'impact hydrodynamique à la côte.

Le site est exposé face à l'Ouest/Nord-Ouest et est soumis frontalement à la direction principale de houle. A l'examen de la disposition du trait de côte et au vu de la régularité des sollicitations hydrodynamiques, le transport sédimentaire principal s'effectue dans le profil.

\section{Matériel et méthodes : modélisation numérique}

3.1 Bathymétrie du site d'étude

Les données bathymétriques suivantes ont été utilisées dans le cadre de l'étude de la houle et de l'impact de la souille :

- La carte SHOM n 6111, donnant la bathymétrie au large ;

- Le levé bathymétrique réalisé en 2013 dans la zone d'étude

La bathymétrie résultante est représentée sur la figure 2 avec un maillage de $5 \mathrm{~m}$. La même bathymétrie en faisant l'hypothèse d'un dragage uniforme de 5 mètres est présentée sur la figure 3 .

La fosse de dragage a une section trapézoïdale avec des pentes de berges de $2.5 \%$.

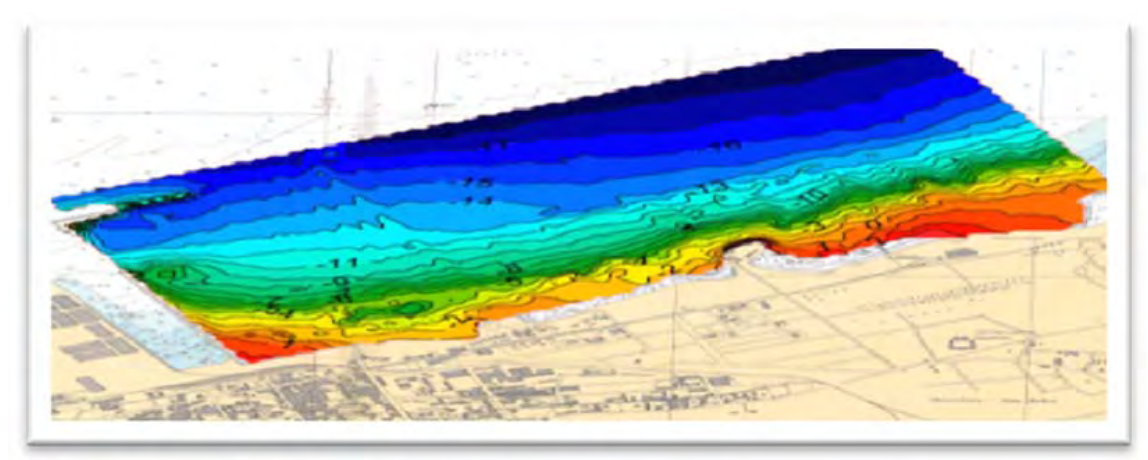

Figure 2. Bathymétrie avant l'extraction de sable.

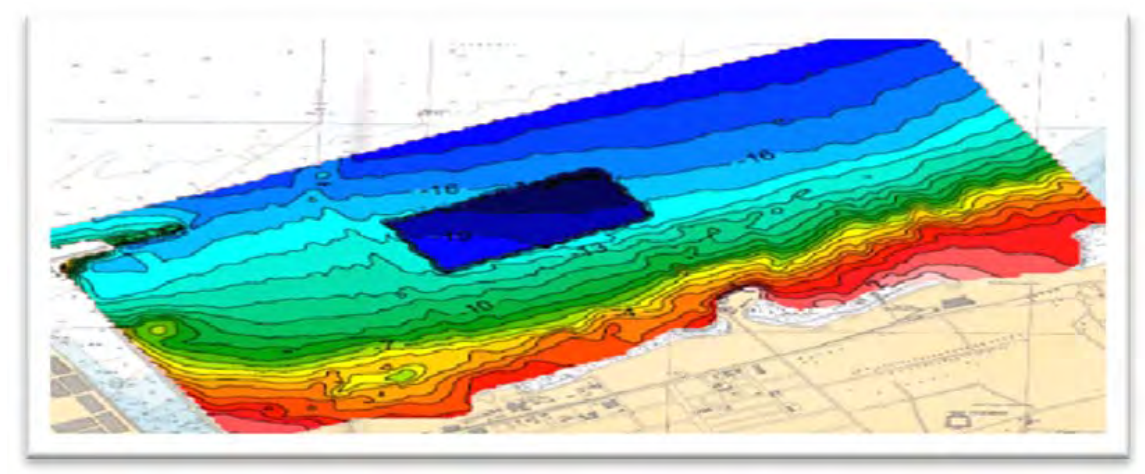

Figure.3. Bathymétrie après l'extraction de sable 


\section{XIV ${ }^{\text {èmes }}$ Journées Nationales Génie Côtier - Génie Civil \\ Toulon, 29 juin au $1^{\text {er }}$ juillet 2016}

\subsection{Paramètres de modélisation:}

Afin de déterminer les conditions de houle au site d'étude, la houle du large a été propagée vers la côte par la mise en œuvre d'un modèle numérique de propagation (modèle SWAN). Ce logiciel représente les phénomènes physiques suivants :

- Propagation de la houle dans le temps et dans l'espace, réfraction dû aux courants et à la profondeur, déplacement de fréquence dû au courant et à la marée ;

- Redistribution de l'énergie de la houle sur le spectre par des interactions non-linéaires comprenant l'interaction de trois et quatre ondes ;

- Frottement sur le fond et déferlement dû à la profondeur et à la cambrure ;

- Surélévation du niveau moyen dû à la houle

Le spectre considéré dans le modèle SWAN est celui de la densité de l'action des vagues (BOOIJ et al., 1999) $\mathrm{N}(\sigma, \theta)$ plutôt que le spectre de la densité d'énergie $E(\sigma, \theta)$.

$x$ et $y$ étant les composantes horizontales de l'espace géographique, $\sigma$ la fréquence relative et $\theta$ la direction.

En coordonnées cartésiennes, cette équation s'écrit sous la forme suivante :

$\frac{\partial}{\partial t} N+\frac{\partial}{\partial x} C_{x} N+\frac{\partial}{\partial y} C_{y} N+\frac{\partial}{\partial \sigma} C_{\sigma} N+\frac{\partial}{\partial \theta} C_{\theta} N=\frac{S}{\sigma} C_{x}, C_{y}, C_{\sigma}, C_{\theta}$ : composantes de la vitesse de propagation obtenues à partir de la théorie linéaire des vagues. Ces coefficients sont donnés explicitement dans le manuel théorique du code de calcul.

\section{Résultats}

Les simulations ont été réalisées par comparaison entre l'état en amont et en aval et l'état sans et avec la souille de 5 mètres. Ces deux états sont présentés sur la figure 4.

Les deux paramètres considérés sont la hauteur et la direction de la houle. Le long de la direction longshore de la souille, on présente sur les figures 5 et 7 une confrontation de la hauteur de la houle entre l'amont et l'aval de la fosse. L'objectif est de quantifier la variation de la hauteur entre les bords de la souille et son milieu. La direction de la houle est de $320^{\circ}$ et son amplitude au large est de 4 mètres. La période est de 12 secondes.

Sur les figures 6 et 8 , on présente l'impact de la souille sur la direction de la houle. On remarque une déviation symétrique des orthogonaux de la houle de l'ordre de 6 à 8 degrés vers l'extérieur de la souille dans les deux cotés (figure 6).

Lors du passage de la houle sur la fosse de dragage sa hauteur subit une diminution de l'ordre de $40 \mathrm{~cm}$ sur $4 \mathrm{~m}$ soit une atténuation d'énergie de la houle de $10 \%$ (figures 5 et 7). La présence de la souille provoque une augmentation du $\mathrm{Hs}$ sur les bords de la zone de dragage. D’une manière générale les simulations font apparaître une diminution du transfert d'énergie de houle au-dessus de la zone d'extraction ce qui provoque un flux d'énergie latéralement à la souille. 


\section{Thème 1 - Hydrodynamique côtière}

En termes d'amplitude de houle, on assiste à une légère diminution à l'aval de la fosse et à une légère augmentation sur ses côtés. L'amplitude à la côte est renforcée de part et d'autre de la souille et est diminuée à son aval direct.

On constate que lorsque la houle aborde la souille, on assiste à une décroissance significative de la vitesse sur le fond et à une faible augmentation de cette même vitesse sur les côtés de la fosse. La souille provoque un transfert d'énergie limité sur les côtés pour compenser la diminution du transfert d'énergie selon la direction de propagation principale de la houle.

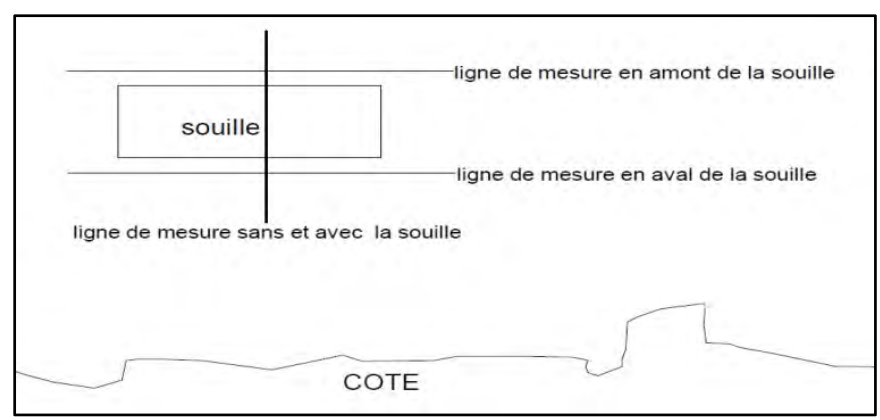

Figure 4. Illustration des lignes de calcul.

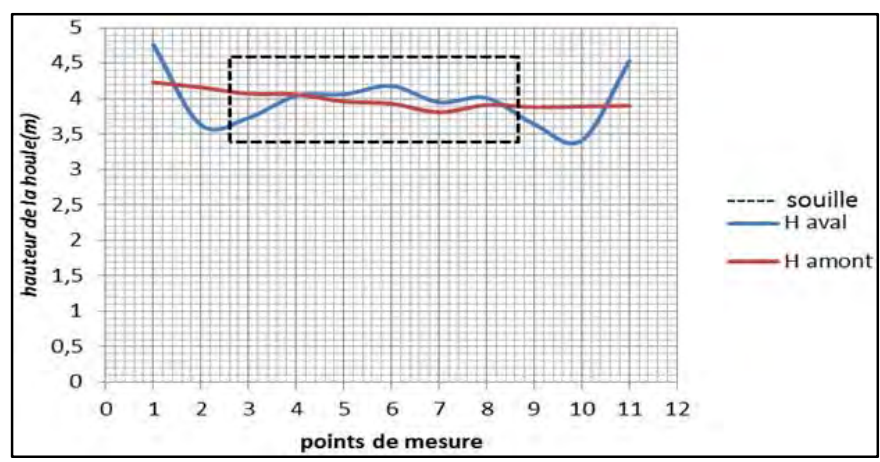

Figure 5. Impact de la souille sur l'évolution de la hauteur Hs sur une ligne de calcul dans la direction longshore en amont et en aval de la souille (fig. 4).

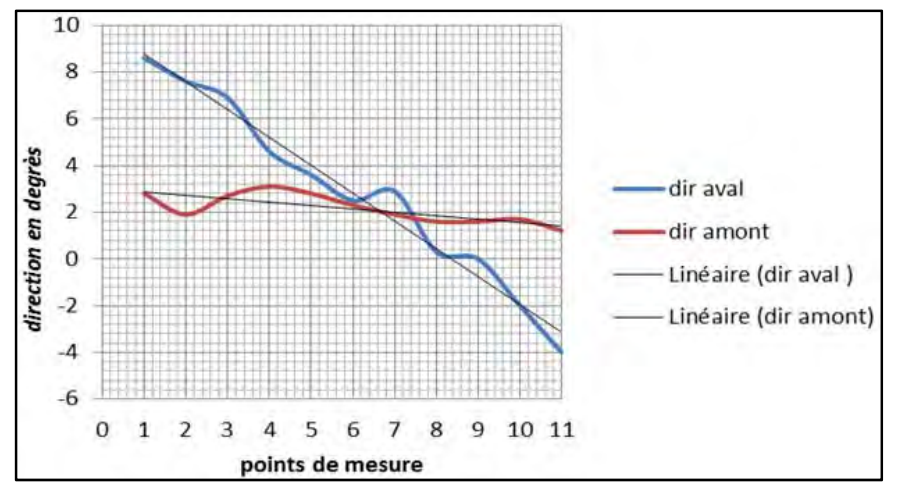

Figure 6. Impact de la souille sur la direction de la houle sur une ligne de calcul dans la direction longshore en amont et en aval de la souille (fig. 4). 


\section{XIV ${ }^{\text {èmes }}$ Journées Nationales Génie Côtier - Génie Civil \\ Toulon, 29 juin au $1^{\text {er }}$ juillet 2016}

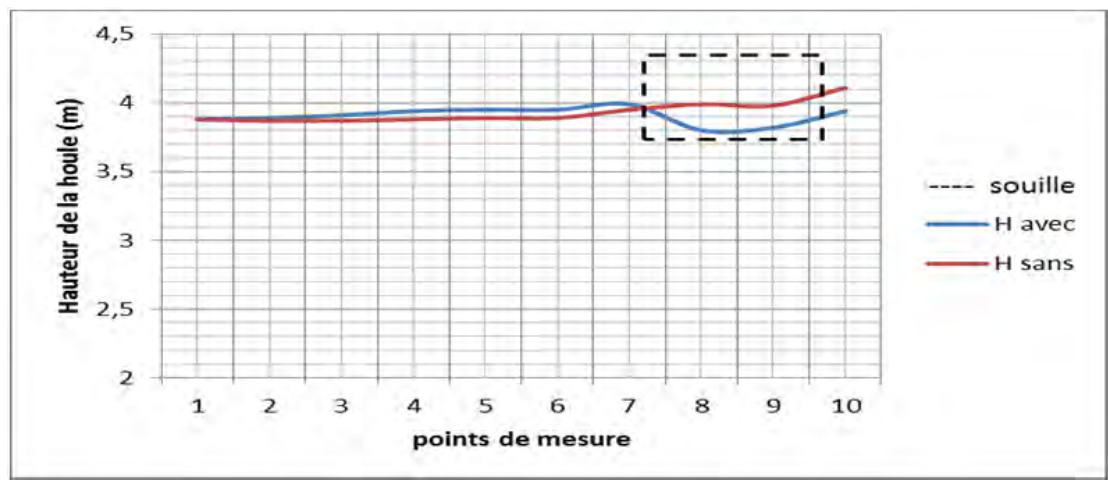

Figure 7. Influence de la souille sur les hauteurs : comparaison des hauteurs $H$ avec et sans souille le long d'une orthogonale de la houle.

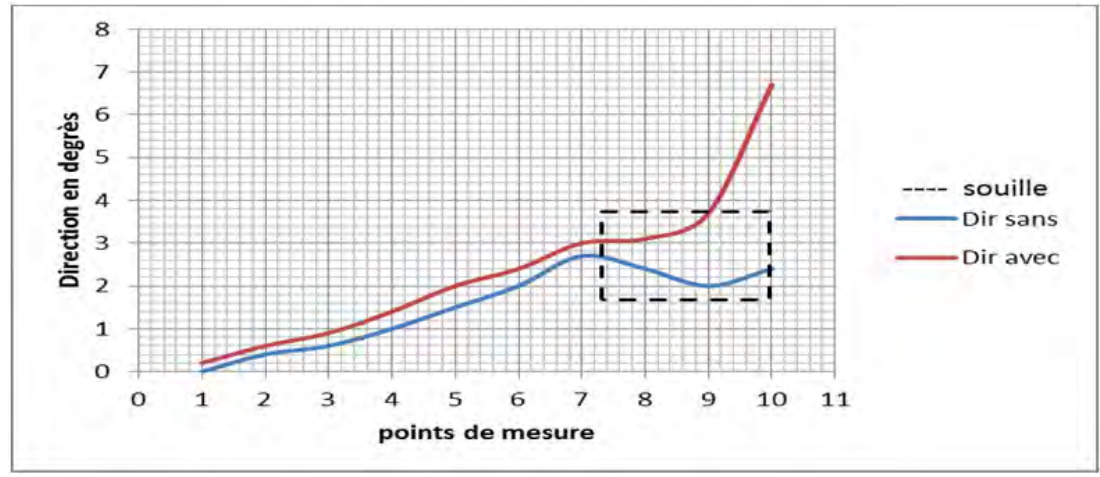

Figure 8. Influence sur la direction : comparaison des directions avec et sans la souille (fig.4).

\section{Conclusions}

Dans ce travail, on a appliqué une méthodologie numérique pour montrer que les fosses de dragage impactent les caractéristiques de la houle. Le site choisi se trouve sur la façade atlantique Marocaine. Les données sont prises dans l'environnement naturel du site : bathymétrie, houle, ...

On retrouve les éléments classiques relatifs à l'altération de la propagation de la houle par la souille de dragage. Le phénomène de réflexion fait qu'on assiste à une diminution de la hauteur de la houle à l'intérieur de la fosse en créant un gradient d'énergie symétrique dans la direction longshore de la souille. Le phénomène de la réfraction se manifeste par une divergence des directions de la houle sur les côtés La méthodologie numérique semble apporter des éléments pour l'étude de l’impact physique du dragage sur la stabilité du littoral.

En perspective, nous allons quantifier cet impact en analysant les cisaillements sur le fond et la tendance de réponse de la côte à l'existence d'une fosse de dragage à proximité de sa ligne. Une autre direction du travail consiste à prendre en considération 
les effets du courant et de raffiner la résolution au voisinage de la fosse en introduisant des modèles imbriqués.

\section{Références bibliographiques.}

BENDER C.J. (2001). Wave field modifications and shoreline response due to offshore borrow areas. MS thesis, Univ. of Florida, Gainesville, Fla.

BOOIJ N.R., RIS R.C., HOLTHUIJSEN L.H. (1999). A thirdgeneration wave model for coastal regions, Part 1, model description and validation. J. Geophys. Res., 104(C4), pp 7649-7666. http://dx.doi.org/10.1029/98JC02622

DEMIR H., OTAY E., WORK P., BÖREKÇI O.S. (2004). Impacts of Dredging on Shoreline Change. Journal Waterway, Port, Coastal, Ocean Engineering, ASCE, Vol. 130(4), pp 170-178. http://dx.doi.org/10.1061/(ASCE)0733-950X(2004)130:4(170)

IDRISSI M., HOURIMECHE A., REY V., CHAGDALI M. (2004). Impact des installations côtières sur l'évolution du littoral de Mohammedia (Maroc). VIII ${ }^{\text {ème }}$ Journées Nationales Génie Côtier - Génie Civil, Compiègne, France, pp 789-796. http://dx.doi.org/10.5150/jngcgc.2004.086-I

KOJIMA H., IJIMA T., NAKAMUTA T. (1986). Impact of offshore dredging on beaches along the Genkai Sea, Japan. Proc. 20th Int. Conf. on Coastal Engineering, ASCE, New York, pp 1281-1295.

MIGNIOT C. (1990). Manuel sur l'hydrodynamique sédimentaire et l'érosion et sédimentation du littoral - $2^{\text {ème }}$ partie: Erosion et sédimentation du littoral. METL, Secrétariat d'état à la mer, STCPMVN, Référence CETMEF : ER PM 88.01. Disponible sur URL : http://www.eau-mer-fleuves.cerema.fr/IMG/pdf/ER_PM_88-02_cle6a4afe-1.pdf MIGNIOT C., VIGUIER J. (1980). Influence de l'extraction des granulats en mer sur l'équilibre $d u$ littoral. La Houille Blanche, $\mathrm{n}^{\circ} \quad 3$, pp 177-194, http://dx.doi.org/10.1051/lhb/1980018

TING C.K.F., RAICHLEN F. (1986). Wave interaction with a rectangular trench. Journal of Waterway. Port, Coastal and Ocean Engineering, ASCE, Vol. 112(3), pp 454-460. http://dx.doi.org/10.1061/(ASCE)0733-950X(1986)112:3(454)

VELEGRAKIS A.F., BALLAY A., POULOS S., RADZEVICIUS R., BELLEC V., MANSO F. (2010). European marine aggregates resources Origins, usage, prospecting and dredging. Journal of Coastal Research SI-51-1-14 West palm Beach Florida Winter. 